\title{
Automatic Hidden Web Database Classification
}

\author{
Zhiguo Gong, Jingbai Zhang, and Qian Liu \\ Faculty of Science and Technology \\ University of Macau \\ Macao, PRC \\ \{fstzgg, ma46597, ma46620\} @umac.mo
}

\begin{abstract}
In this paper, a method for automatic classification of Hidden-Web databases is addressed. In our approach, the classification tree for Hidden Web databases is constructed by tailoring the well accepted classification tree of DMOZ Directory. Then the feature for each class is extracted from randomly selected Web documents in the corresponding category. For each Web database, query terms are selected from the class features based on their weights. A hidden-web database is then probed by analyzing the results of the class-specific query. To raise the performance further, we also use Web pages which have links pointing to the hidden-web database (HW-DB) as another important source to represent the database. We combine link-based evaluation and query-based probing as our final classification solution. The experiment shows that the combined method can produce much better performance for classification of hidden Web Databases.
\end{abstract}

\section{Introduction}

With the explosive growth of the World Wide Web, the traditional Crawlers fail to satisfy the users' demand for information searching yet. Many recent studies $[1,2]$ have observed that a significant fraction of Web content known as the Hidden-Web (HW) [3], the Invisible Web [4], or the Deep Web [2], lies outside the PIW. In fact, these pages can only be dynamically generated in response to users' queries, which the traditional Crawlers cannot handle. However, we cannot simply ignore them, because some recent studies claim that the size of the Hidden-Web pages are as many as 500 billion pages, comparing to "only" two billion pages of the ordinary web [5]. Furthermore, the information on the $\mathrm{HW}$ is usually generated from structured databases, which are referred to as Hidden-Web Databases (HW-DB) [6]. In [7], the study has estimated that there are 250,000 private databases, and the access of $95 \%$ of them is free. These databases represent $54 \%$ of the Hidden Web.

In this paper, in order to effectively guide users to find the relevant information from such databases, we present a prototype system for classifying the HW-DB into a predefined category hierarchy which is tailored from some existing classification tree for Web documents. The feature for each class is extracted from randomly selected Web documents in corresponding Web class. For each Web database, query terms are selected from such class features based on their weights. A hidden-web database is 
then probed by analyzing the results of the class-specific query to the hidden database. To raise the performance further, we also use Web pages which have links pointing to the hidden database as another important source to represent the database. We combine link-based evaluation and query-based probing as our final classification solution for hidden database classification. In addition, our focus is on text databases, since $84 \%$ of all searchable databases on the web are estimated to provide access to text documents [2], and other kinds of databases like image or video databases are out of the scope of this paper.

The contributions presented in this article are organized as follows. We present the details of our HW-DB classification system based on query probing and based on link evaluation in Section 2. A system evaluation is conducted and important experimental results are discussed in Section 3. And finally section 4 provides conclusions.

\section{Hidden Databases Classification}

Our system aims to automatically assign each Hidden-Web Database to the "best" category or categories of the classification scheme. Instead of constructing a new classification scheme manually, similar to the approaches proposed by $[5,7]$, we exploit a category hierarchy for HW-DB classification from the popular DMOZ Directory. Fig.1. shows a fraction of the category hierarchy used in our system.

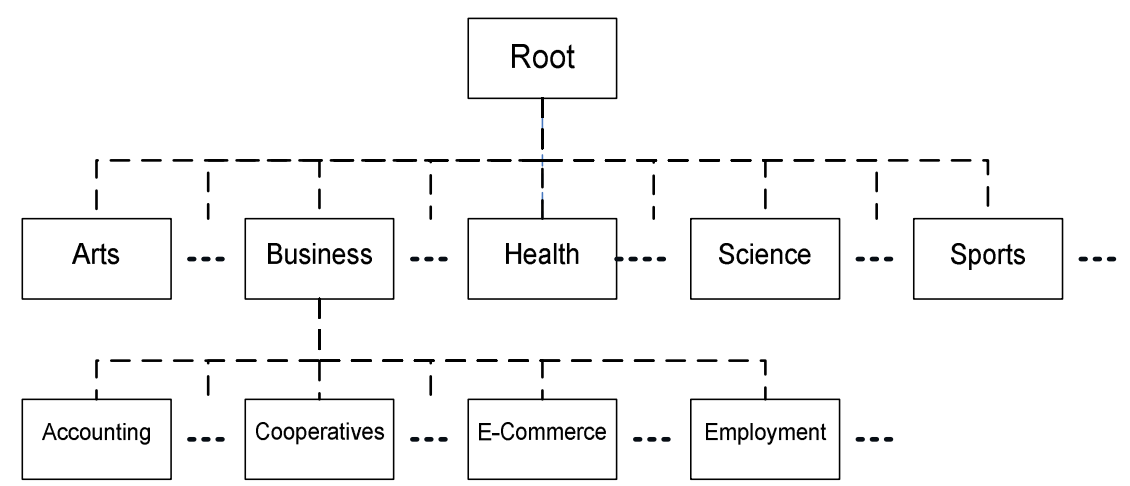

Fig. 1. A fraction of the category hierarchy for HW-DB used in our system

\subsection{Classification Models}

In order to assign a Web document to corresponding categories, a classifier algorithm is needed. Several classifier models exist in literature, such as SVM (Support Vector Machine) [8], kNN (key nearest Neighbor) [9], LLSF (Linear Least Square Fit) [10], NNet (Neural Network) [11], NB (Naïve Bayes) [11], and RIPPER [12]. Though [6] shows that RIPPER can provide good overall performance for HW-DB classification, the correctness of the rules for each category are critical for the precision of the classifications. However, it is a hard work for correct rule extractions. Take the rule ("ibm" AND "computer" $\rightarrow$ "Computer") as an example, even though some document 
may contain both "ibm" and "computer", it may not belong to the category of "Computer" in many cases. Furthermore, the classification needs to extensively interact with a HW-DB. That means, for each rule the system needs to interact at least one time with the database. Y. Yang and X. Liu compared other classifiers and pointed out that model SVM, kNN and NB can always produce better performance for the document classifications over LLSF and NNet. Considering both effectiveness and efficiency as the important factors, in our work, we employ kNN as the classifier for HW-DB classifications.

To use $\mathrm{kNN}$, training documents for each category are needed. In our implementation, for any category $\boldsymbol{c}_{\boldsymbol{i}}$, we select $N$ Web documents $\left(d_{j, 1}, d_{j, 2}, \ldots, d_{j, N}\right)$ from the corresponding DMOZ directory. Then, for any Web document $\boldsymbol{x}$, the classification rule in $\mathrm{kNN}$ can be written as:

$$
\operatorname{Similarity}\left(x, c_{j}\right)=\sum_{1 \leq i \leq N} \operatorname{sim}\left(x, d_{j, i}\right)-b_{j}
$$

where $\operatorname{sim}\left(x, d_{j, i}\right)$ is the metric of similarity between $x$ and $d_{j, i}, b_{j}$ is the category specific threshold for the binary classification.

In order to calculate $\operatorname{sim}\left(x, d_{j, i}\right)$, we represent each Web document $d$ as a vector $\left(w_{1}, w_{2}, \ldots w_{M}\right)$, where $w_{l}$ is the weight of term $t_{l}$ in $d$. And $w_{l}$ is defined as:

$$
w_{l}=\frac{t f\left(t_{l}, d\right)}{\operatorname{Max}_{\{n\}}\left\{t f\left(t_{n}, d\right)\right\}}
$$

where $t f\left(t_{l}, d\right)$ is the frequency of term $t_{l}$ in $d$. With this definition, for any two web documents $d=\left(w_{1}, w_{2}, \ldots w_{M}\right)$ and $d^{\prime}=\left(w^{\prime}{ }_{1}, w_{2}{ }_{2}, \ldots w^{\prime}{ }_{M}\right), \operatorname{sim}\left(d, d^{\prime}\right)$ is defined as the cosine value between them:

$$
\operatorname{sim}\left(d, d^{\prime}\right)=\frac{\sum w_{l} * w^{\prime}{ }_{l}}{\sqrt{\sum\left(w_{l}\right)^{2}} \sqrt{\sum\left(w^{\prime}{ }_{l}\right)^{2}}} .
$$

The original kNN classifier is designed for document classification. For a hidden Web database $H D$, let $\left\{h d_{1}, h d_{2}, \ldots, h d_{R}\right\}$ be all the documents contained in $H D$. We concatenate all the documents of $H D$ into one document, still denoted as $H D$. That is, $H D=\cup h d_{j}$. Then, $H D$ is assigned to category $c$ if and only if $\operatorname{Similarity}(H D, c)>0$. However, we do not have the knowledge about the documents within $H D$. To solve the problem, we use two techniques to approximate it in this study. Firstly, we detect the HW-DB through probing; secondly, link structure of the Web is used.

\subsection{Hidden Database Probing}

In each category, some queries are needed for probing hidden databases. [6] uses extensive number of rules or queries for probing. As mentioned before, multiple query probing is expensive for both rule extracting and database probing. For such reasons, in our approach, we only use one query for probing in each category. Our one-query probing is based on the assumption that it does not affect the classification too much because every category uses the same number of queries (one query in our paper). We extract candidate query terms for each category from the concatenation of its all training documents selected from the corresponding DMOZ Directory. Those 
terms, called category feature, are ordered with their weights. We chose several terms according to their weights as a query to probe hidden databases.

After sending the request message including form filled-out information to the server, our proposed system will receive the result pages. Perhaps the most common case is that a web server returns results page by page consecutively, with a fixed number, say ten or twenty, result matches per page.

To classify the HW databases effectively, we need to analyze the content of each result document. However, full-text of results from some HW-DB cannot be obtained for some reasons like copyright. So the system handles differently for these two situations.

\subsubsection{Result Documents Without Full-Text}

In this situation, only the number of returned documents for the query can be used for analysis. Let $c_{1}, c_{2}, \ldots, c_{K}$ be $K$ categories under the same parent node in the hierarchy, the returned number for each category by a hidden database $H D$ are $L_{l}, L_{2}$, $\ldots, L_{K}$ respectively. Then, we approximate $H D$ as:

$$
H D \approx \sum_{1 \leq j \leq K} f_{j} * L_{j}
$$

where $f_{j}$ is the category feature of class $\boldsymbol{c}_{\boldsymbol{j}}$. In fact, $f_{j}$ is the centroid of the training documents in $c_{j}$.

\subsubsection{Result Documents with Full-Text}

For the hidden databases whose full-texts can be accessed, our system can analyze the document content further to get more accurate approximation for $H D$. In such case, not only the number of the results for a category can be got, but also the relevance of the documents can be used. To save the cost, we only access documents in several positions along the result list. For example, the positions can be set to the first result and the last result, or more complex to $0 \%, 25 \%, 50 \%, 75 \%$, and $100 \%$ of the result list.

Suppose we only access the first document $h d_{j, 1}$ and the last document $h d_{j, L j}$ for category $\boldsymbol{c}_{j}$. Then, the hidden database $H D$ can be approximated as:

$$
H D \approx \sum_{1 \leq j \leq K} \frac{1}{2}\left(h d_{j, 1}+h d_{j, L_{j}}\right) * L_{j}
$$

where $L_{j}$ is defined as before. It can be easily extended to support more document accesses.

With the probed result for a hidden database $H D$ (equation 4, or 5), $H D$ can always be classified using equation (1).

\subsection{HW-DB Classification Based on Link Structure}

In last subsection, we introduced the methods for the Hidden-Web databases classification based on probing, which produces good experimental results. However, it does not make use of the properties of Web structure, especially the links among the Web documents. Actually, link structure of the Web provides another important clue 
for HW-DB classifications. In fact, as in fig.2, a hidden database may be referenced by many Web pages. Those pages can also be used to derive the semantics of the hidden database.

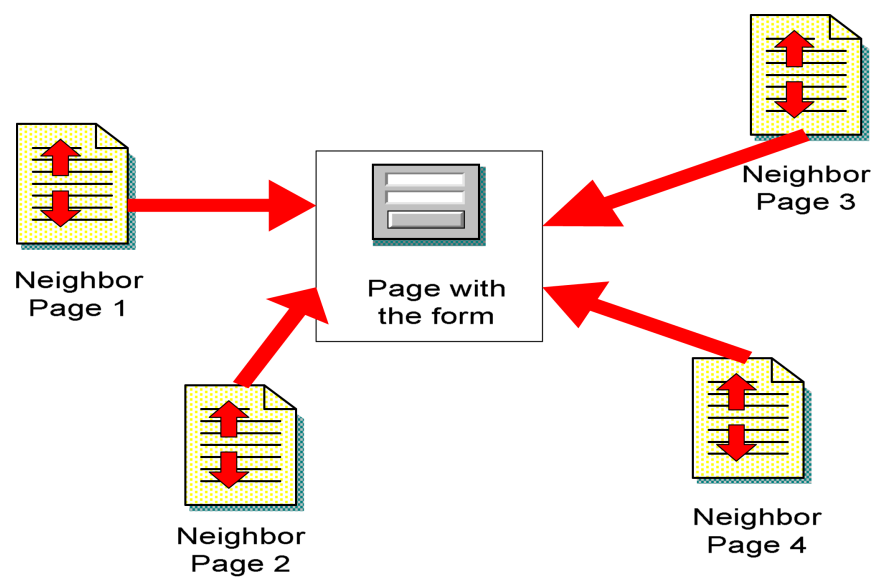

Fig. 2. A Hidden database linked by other pages (neighbor pages)

Web pages, which have links to the hidden database $H D$, are called neighbor pages for this database. To use them for the classification of $H D$, we concatenate all of the neighbor pages into one document called $N P$. Then, the semantics of $H D$ is represented with a vector of terms extracted from $N P$. Therefore, $H D$ is also can be classified by the values of Similarity $(N P, c)$.

\subsection{Combined Classifier for Hidden Databases}

To raise the performance of the classification, we try to combine probing model with link-based model. In fact, new hidden databases often have less neighbor pages to be referenced. Therefore, probing method is the only way for the classification in such situation. To avoid outlier, we use link-based classifiers only for hidden databases which have at least 20 neighbor pages. The combined classifier is defined as:

$$
C \text {-Similarity }(H D, c)=W^{*} \operatorname{Similarity}(H D, c)+(1-W) * \operatorname{Similarity}(N P, c)
$$

where $W$ is used to balance this two classifiers.

\section{Experiment}

Our objective functions for system performance are based on two basic metricsprecision and recall [5].

When evaluating the result of classification, there are three important values for each category:

A ---- Number of documents which are classified into the category correctly;

B ---- Number of documents which are classified into the category wrongly; 


\section{C ---- Number of documents which are classified into other category wrongly;}

Recall is the ratio of the number of documents classified into a category correctly to the total number of relevant documents in the same category. Precision is the ratio of the number of documents classified into a category correctly to the total number of irrelevant and relevant documents classified into the same category. Both of them can be represented with the above values, $\mathrm{A}, \mathrm{B}$, and $\mathrm{C}$.

$$
\text { precision }=\frac{A}{A+B} \times 100 \% \quad, \text { recall }=\frac{A}{A+C} \times 100 \%
$$

To condense precision and recall into one number, we use the $\mathrm{F}_{1}$-measure metric [5]:

$$
F_{1}=\frac{2 \times \text { precision } \times \text { recall }}{\text { precision }+ \text { recall }}
$$

which is only high when both precision and recall are high, and is low for design options that trivially obtain high precision by sacrificing recall or vice versa. Recall and precision are evenly weighted.

\subsection{Determining the Number of the Feature Terms for Form Filling-Out}

There are two types of form-elements in general, A-Element (support Boolean 'AND') and O-Element (support Boolean 'OR'). We must choose a proper number of query terms for filling out form elements for these two types. A-Element and OElement models occupy $41 \%$ and $59 \%$ respectively in our testing hidden databases. We fill-out those hidden databases with changing number of query terms.

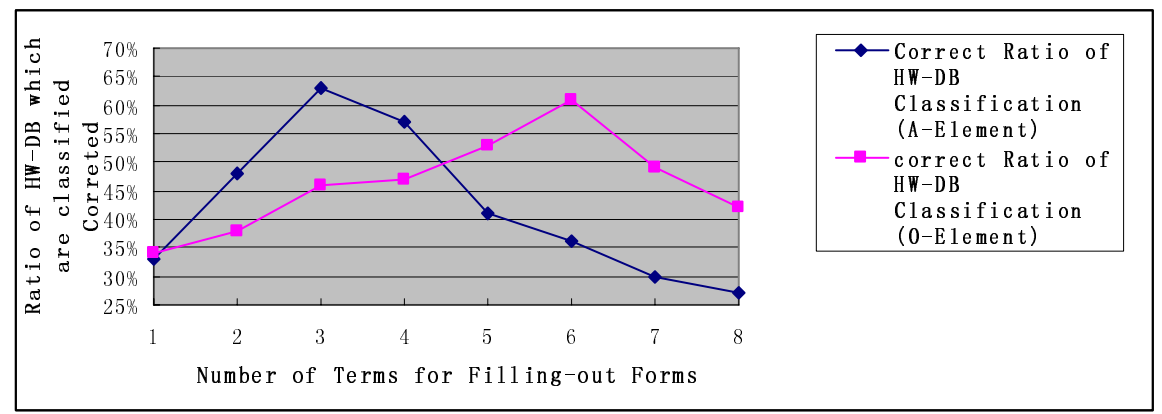

Fig. 3. The ratio of HW-DB which are classified correctly using different quantities of terms

Fig.3. shows the correct ratio of HW-DB classification using different numbers of feature terms. The horizontal axis shows the number of terms to fill-out the forms and the vertical axis shows the ratio of HW-DB which are classified correctly. It can be seen from the figure, for A-Element, the correct radio reaches its summit $63 \%$ when we choose 3 terms to fill-out the forms. For O-Element, the optimal number of terms is 6 , which leads to $61 \%$ correct radio. That is, we should choose 6 terms to fill-out the forms for O-Element, in order to receive the maximal correct ratio. 


\subsection{Evaluating Results over Different Classification Approaches}

In our system, three basic models for classification of hidden databases are addressed, including full-text probing $\mathrm{M}_{1}$, result-number only probing $\mathrm{M}_{2}$, and link-based classifying $\mathrm{M}_{3}$. By combining $\mathrm{M}_{1}$ with $\mathrm{M}_{3}, \mathrm{M}_{2}$ and $\mathrm{M}_{3}$, we get two combined classification models.

Fig.4. shows the classification performances for basic model $\mathrm{M}_{1}$ and $\mathrm{M}_{2}$, as well as the two combined models. It is clear from the figure, by far the combined method $\left(M_{1}\right.$ $+M_{3}$ ) receives the best performance when balance perimeter $W=0.4$. And the second combined mode $\left(M_{2}+M_{3}\right)$ reaches its optimal performance when $W=0.3$.

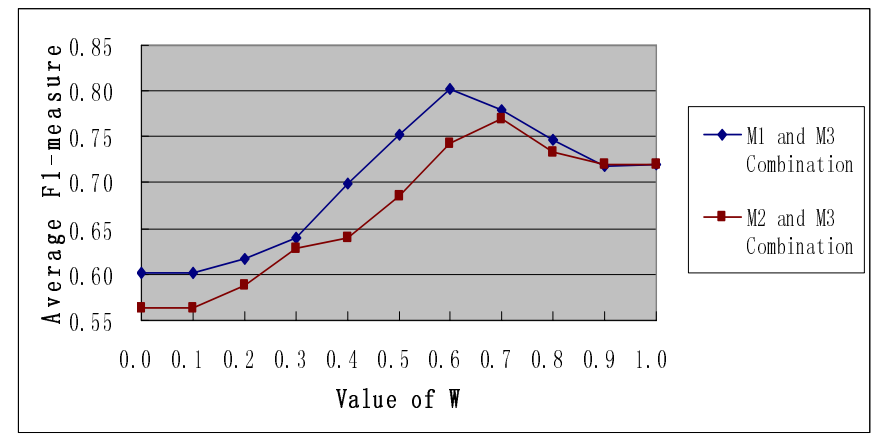

Fig. 4. The Classification Performance of Combine Methods by Different Weight

The average $F_{1}$-measures of those methods are shown in Table 1 . By far, the combined method $\left(M_{1}+M_{3}\right)$ is the best approach for HW-DB classification. However, other methods should not be abandoned since each method has its own merit. Method $\mathrm{M}_{1}$ and $\mathrm{M}_{3}$ are the basic ones for the combine method $\left(M_{1}+M_{3}\right)$. Although method $M_{2}$ shows the worst performance among them, it is a good alternative if a HW-DB cannot returns full-text of result documents. In addition, $M_{2}$ is with the low cost comparing with $M_{l}$.

Table 1. Average $F_{1}$-measure of $M_{1}, M_{2}, M_{3}$ and the Combine Methods

\begin{tabular}{|c|c|c|c|c|}
\hline Methods & $M_{1}$ & $M_{2}$ & $M_{2}+M_{3}$ & $M_{1}+M_{3}$ \\
\hline $\begin{array}{c}\text { Average } \mathrm{F}_{1^{-}} \\
\text {measure }\end{array}$ & 0.60 & 0.56 & 0.72 & 0.80 \\
\hline
\end{tabular}

\section{Conclusions}

In this paper, we have proposed a novel and efficient approach for classification of Hidden-Web Databases. We have introduced a category hierarchy for HW-DB and described the process to extract the feature for each category. With terms of the features, we probe the hidden databases and analyze the results documents in order to 
classify the HW-DB. To raise the performance further, we also use Web pages which have links pointing to the hidden-web database as another important source to represent the databases. We combine link-based evaluation and query-based probing as our final classification solution. Our experiment shows the combined approach can generate a much better performance for the HW-DB classification.

Acknowledgement. This Work was supported in part by the University Research Committee under Grant No. RG069/05-06S/07R/GZG/FST and by the Science and Technology Development Found of Macao Government under Grant No. 044/2006/A.

\section{References}

[1] Lawrence, S., Giles, C.L.: Accessibility of Information on the Web. Nature 400, 107-109 (1999)

[2] Bergman, M.K.: The Deep Web: Surfacing Hidden Value Latest Access: 11/1/2007 (September 2001), http://www.brightplanet.com/resources/details/deepweb.html

[3] Raghavan, S., Garcia-Molina, H.: Crawling the Hidden Web. In: Proceedings of the 27th International Conference on Very Large Data Bases (VLDB) (2001)

[4] Lin, K.I., Cheng, H.: Automatic Information Discovery form the Invisible Web. In: Proceedings of the International Conference on Information Technology: Coding and Computing (ITCC) (2002)

[5] Ipeirotis, P.G., Gravano, L., Sahami, M.: Probe, Count, and Classify: Categorizing Hidden-Web Databases. In: Proceedings of the 20th ACM SIGMOD International Conference on Management of Data, ACM Press, New York (2001)

[6] Gravano, L., Ipeirotis, P.G., Sahami, M.: QProber: A System for Automatic Classification of Hidden-Web Databases. ACM Transactions on Information Systems (TOIS) 21(1), 141 (2003)

[7] Bergholz, A., Chidlovskii, B.: Crawling for Domain-Specific Hidden Web Resources. In: Proceedings of the 4th International Conference on Web Information Systems Engineering (WISE '03) (2003)

[8] Vapnik, V.: The Nature of Statistic Learning Theory. Springer, New York (1995)

[9] Dasarathy, B.V.: Nearest Neighbor (NN) Norms: NN Pattern Classification Techniques. In: McGraw-Hill Computer Science Series, IEEE Computer Society Press, Las Alamitos, California (1991)

[10] Yang, Y., Chute, C.G.: An example-based mapping method for text categorization and retrieval. ACM Transaction on Information Systems (TOIS) 12(3), 252-277 (1994)

[11] Mitchell, T.: Machine Learning. McGraw Hill, New York (1996)

[12] Cohen, W.W.: Learning trees and rules with set-valued features. In: Proceedings of the Thirteenth National Conference on Artificial Intelligence, pp. 709-716 (1996) 